\title{
ON VARIOUS TYPES OF SHADOWING FOR GEOMETRIC LORENZ FLOWS
}

\author{
A. ARBIETO, J.E. REIS AND R. RIBEIRO
}

\begin{abstract}
We show that Lorenz flows have neither limit shadowing property nor average shadowing property nor the asymptotic average shadowing property where the reparametrizations related to these concepts relies on the set of increasing homeomorphisms with bounded variation.
\end{abstract}

1. Introduction. The shadowing property is a dynamical property that plays a key role in the study of the stability of dynamics. This property is found in hyperbolic dynamics, and it was used with success to prove their stability, see for instance [12]. Roughly speaking, it allows us to trace a set of points which looks like an orbit, but with errors, by a true orbit. For practical applications, we can suppose that $\varphi$ is viewed as the orbit realized in numerical calculation by computer, or in physical experiments; thus, it could have errors. Then the shadowing property allows us to "correct" these errors, finding a true evolution which nicely approximates $\varphi$. Thus, to decide which systems possess the shadowing property is an important problem in dynamics.

The geometric Lorenz model is an important example in the theory of dynamical systems. It was inspired by the equations found by Lorenz related to a model of fluid convection [10]. Moreover, it is one of the most famous examples, since it is often related with the notion of chaos. It was studied in the initial stages by Guckenheimer and Williams [7, 14, 15], Afraimovich, Bykov and Shil'nikov [1] and Yorke and Yorke [16]. Moreover, it is an attractor which is transitive

2010 AMS Mathematics subject classification. Primary 37C50, 37D45, Secondary $37 \mathrm{C} 10$.

Keywords and phrases. Average shadowing, asymptotic average shadowing, limit shadowing, geometrical Lorenz flows.

The first author was partially supported by CNPq, FAPERJ and PRONEX/DS from Brazil. The second author was supported by CAPES. The third author was supported by CNPq.

Received by the editors on June 27, 2012, and in revised form on July 7, 2013. 
and contains both regular orbits and singularities. As we mentioned before, hyperbolic dynamical systems possess the shadowing property. However, these Lorenz systems are not hyperbolic, since they have singularities approximated by regular orbits. Even so, they have some robust properties which are also shared by hyperbolic dynamics.

It is natural then to ask if these Lorenz systems have the shadowing property. Komuro [9] showed that geometric Lorenz flows do not satisfy the (parameter-fixed) shadowing property except in very restricted cases. Even so, in [8], it was shown that the geometric Lorenz attractors have the parameter-shifted shadowing property. However, this notion is very technical. So, we could ask if these systems have some shadowingtype properties which are more easy to check.

Related to this, many properties were suggested and studied by several authors. As a kind of generalization of the shadowing property, Blank [3] introduced the notion of the average shadowing property in the study of chaotic dynamical systems. Essentially it allows great errors, but they must be compensated with small errors.

On the other hand, Eirola et al. [4] posed the notion of the limitshadowing property. From the numerical point of view this property on a dynamical system $X$ means that if we apply a numerical method of approximation to $X$ with "improving accuracy" so that one step errors tend to zero as time goes to infinity, then the numerically obtained trajectories tend to real ones. Such situations arise, for example, when one is not so interested in the initial (transient) behavior of trajectories but wants to reach areas where "interesting things" happen (e.g., attractors) and then improve accuracy. In the sequence, Gu [5] introduced the notion of asymptotic average shadowing property for flows. This is a certain generalization of the limit-shadowing property in random dynamical systems.

It could be checked that these weaker shadowing properties are present in hyperbolic dynamics. Thus, following Komuro, a natural question is to decide if the Lorenz systems has some of these weaker shadowing properties. We remark also, as is well known, that the analysis of shadowing on flows becomes more complicated than the analysis for diffeomorphisms, due to the presence of reparametrizations of the systems on those concepts.

The purpose of this paper is to seek sufficient conditions over the 
Lorenz map which implies these kinds of shadowing: $\Delta$-asymptotic average shadowing property $(\Delta$-AASP $), \Delta$-limit shadowing property $(\Delta$-LSP $)$ and $\Delta$-average shadowing property $(\Delta$-ASP $)$. The constant $\Delta$ is an upper bound to the variation of the respective reparametrizations (see Section 2).

Let $(\Lambda, \varphi)$ be a geometric Lorenz flow with Poincaré map $P: S^{*} \rightarrow S$ (see the definitions in Section 2). Let $f: \mathcal{F}^{*} \rightarrow \mathcal{F}$ be the map associated to the foliation $\mathcal{F}$ on $S$ and $L^{+}$and $L^{-}$the lateral leaves of $\mathcal{F}$. Our main result is the following:

Theorem 1.1. Lorenz flows $(\Lambda, \varphi)$ satisfying $f\left(L^{+}\right) \neq L^{+}$or $f\left(L^{-}\right) \neq$ $L^{-}$have neither $\Delta$-ASP, $\Delta$-LSP nor $\Delta$-AASP for any $\Delta \geq 0$.

This paper is organized as follows: in Sections 2 and 3 we give the precise definitions of the objects used in the statement above. In Section 4, we prove Theorem 1.1.

2. Various types of shadowing. There are several types of shadowing in the literature. In this section, we define the ones which will be worked in this paper.

Let

Rep $=\{g: \mathbb{R} \longrightarrow \mathbb{R}: g$ is a monotone increasing homeomorphism with $g(0)=0\}$.

Fixing $\Delta \in R^{+}$, define

$\operatorname{Rep}(\Delta)=\left\{g \in \operatorname{Rep}:\left|\frac{g(s)-g(t)}{s-t}-1\right| \leq \Delta\right.$, for every $\left.s \neq t s, t \in \mathbb{R}\right\}$.

Let $X \in \mathfrak{X}^{1}(M)$ be a vector field and $\varphi$ the associated flow. A sequence $\left(x_{i}, t_{i}\right)_{i \in \mathbb{Z}}$ is a $\delta$-average-pseudo orbit of $\varphi$ if $t_{i} \geq 1$ for all $i \in \mathbb{Z}$ and there is a positive integer $N$ such that for any $n \geq N$ and $k \in \mathbb{Z}$, we have

$$
\frac{1}{n} \sum_{i=1}^{n} d\left(\varphi\left(t_{i+k}, x_{i+k}\right), x_{i+k+1}\right)<\delta
$$


A $\delta$-average-pseudo orbit, $\left(x_{i}, t_{i}\right)_{i \in \mathbb{Z}}$, of $\varphi$ is $\Delta$-positively $\epsilon$-shadowed on average by the orbit of $\varphi$ through $x$, if there exists $h \in \operatorname{Rep}(\Delta)$ such that

$$
\limsup _{n \rightarrow \infty} \frac{1}{n} \sum_{i=1}^{n} \int_{s_{i}}^{s_{i+1}} d\left(\varphi(h(t), x), \varphi\left(t-s_{i}, x_{i}\right)\right) d t<\epsilon,
$$

where $s_{0}=0$ and $s_{n}=\sum_{i=0}^{n-1} t_{i}, n \in \mathbb{N}$. It is $\Delta$-negatively $\epsilon$-shadowed on average by the orbit of $\varphi$ through $x$ if there is $\widetilde{h} \in \operatorname{Rep}(\Delta)$ for which the limit (2.1) is true when replacing $h$ by $\widetilde{h}$ and the limits of integration by $-s_{-i}$ and $-s_{-i+1}$ (in this case $s_{-n}=\sum_{i=-n}^{-1} t_{i}$ ).

Definition 2.1. The flow $\varphi$ has the $\Delta$-average shadowing property $(\Delta$ ASP) if, for any $\epsilon>0$, there exists $\delta>0$ such that any $\delta$-average-pseudo orbit of $\varphi$ is both $\Delta$-positively (negatively) $\epsilon$-shadowed in average by some orbit of $\varphi$.

A sequence $\left(x_{i}, t_{i}\right)_{i \in \mathbb{Z}}$ is a limit-pseudo orbit of $\varphi$ if $t_{i}>1$ for all $i \in \mathbb{Z}$ and

$$
\lim _{|i| \rightarrow \infty} d\left(\varphi\left(t_{i}, x_{i}\right), x_{i+1}\right)=0 .
$$

A limit-pseudo orbit, $\left(x_{i}, t_{i}\right)_{i \in \mathbb{Z}}$, of $\varphi$ is $\Delta$-positively shadowed in limit by an orbit of $\varphi$ through $x$ if there is $h \in \operatorname{Rep}(\Delta)$ such that

$$
\lim _{i \rightarrow \infty} \int_{s_{i}}^{s_{i+1}} d\left(\varphi(h(t), x), \varphi\left(t-s_{i}, x_{i}\right)\right) d t=0 .
$$

Analogously, as before, we define when a limit-pseudo orbit is $\Delta$ negatively shadowed in limit by an orbit.

Definition 2.2. The flow $\varphi$ has the $\Delta$-limit shadowing property $(\Delta$ LSP) if every limit-pseudo orbit is both $\Delta$-positively (negatively) shadowed in limit by an orbit of $\varphi$.

A sequence $\left(x_{i}, t_{i}\right)_{i \in \mathbb{Z}}$ is an asymptotic average-pseudo orbit of $\varphi$ if $t_{i} \geq 1$ for all $i \in \mathbb{Z}$ and

$$
\lim _{n \rightarrow \infty} \frac{1}{n} \sum_{i=-n}^{n} d\left(\varphi\left(t_{i}, x_{i}\right), x_{i+1}\right)=0 .
$$


An asymptotic average-pseudo orbit, $\left(x_{i}, t_{i}\right)_{i \in \mathbb{Z}}$, of $\varphi$ is $\Delta$-positively asymptotically shadowed in average by an orbit of $\varphi$ through $x$ if there exists $h \in \operatorname{Rep}(\Delta)$ such that

$$
\lim _{n \rightarrow \infty} \frac{1}{n} \sum_{i=0}^{n} \int_{s_{i}}^{s_{i+1}} d\left(\varphi(h(t), x), \varphi\left(t-s_{i}, x_{i}\right)\right) d t=0 .
$$

Similarly, we define when an asymptotic average-pseudo orbit is $\Delta$ negatively asymptotic shadowed in average by an orbit.

Definition 2.3. The flow $\varphi$ has the $\Delta$-asymptotic average shadowing property ( $\Delta$-AASP) if every asymptotic average-pseudo orbit is both $\Delta$-positively (negatively) asymptotically shadowed in average by an orbit of $\varphi$.

\section{Geometric Lorenz flows.}

3.1. Construction. Let $S^{3}=\mathbb{R}^{3} \cup\{\infty\}$ be the 3 -sphere. The geometric Lorenz attractor is an attractor set in $S^{3}$ of a flow denoted by $Y_{t}$ that we are about to describe. This attractor has, as a local basin, a solid 2-torus $U$ in $\mathbb{R}^{3}$ such that the flow $Y_{t}$ is inwardly transverse to the boundary of $U$. In $S^{3} \backslash U$, the flow $Y_{t}$ has three saddle type hyperbolic singularities with stable complex eigenvalues and a source at $\{\infty\}$. Define

$$
\Lambda=\bigcap_{t \geq 0} Y_{t}(U)
$$

as the maximal $Y_{t}$-invariant set in $U$. The set $\Lambda$ is called the geometric Lorenz attractor. See Figure 1(b). This geometric model is motivated by Lorenz's equations:

$$
X(x, y, z)=(-a x+a y, r x-y-x z, x y-b z), \quad a, r, b>0,
$$

which were the result of tentatively modeling the weather forecast in the sixties (1963). When the parameters in (3.1) are $a=10, r=28$ and $b=8 / 3$, then the numeric simulation of this field exhibits a behavior which is similar to the field $Y$ called the geometric Lorenz model, which was introduced by Guckenheimer [7, 1976] and by Shilnikov [1]. To understand this geometric model first consider the flow $X_{t}$ associated to the Lorenz field near the origin $O=(0,0,0)$. Analogously, the field 


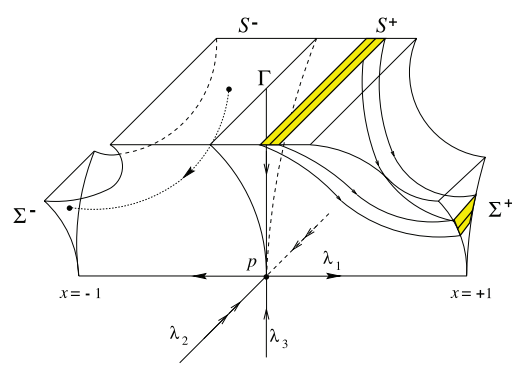

(a) Behavior near the origin.

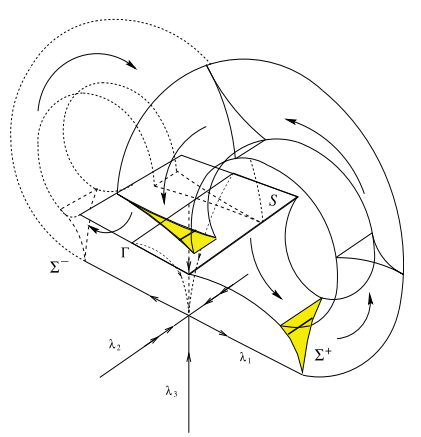

(b) The flow takes $\Sigma^{ \pm}$to $S$.

FigurE 1. Construction of the geometric Lorenz flow.

$Y$ has a hyperbolic singularity at $O$ and, by the Hartman-Grobman theorem, it is conjugated to the linearized equations in a neighborhood of the origin

$$
x^{\prime}=\lambda_{1} x, \quad y^{\prime}=\lambda_{2} y, \quad z^{\prime}=\lambda_{3} z .
$$

Solving this system with initial data $(x(0), y(0), z(0))=\left(x_{0}, y_{0}, 1\right)$, we have:

$$
x(t)=x_{0}\left(e^{t}\right)^{\lambda_{1}}, \quad y(t)=y_{0}\left(e^{t}\right)^{\lambda_{2}}, \quad z(t)=\left(e^{t}\right)^{\lambda_{3}} .
$$

Fix $x_{0}>0$, and let $T$ be the positive time for which the orbit intersects the plane $x=1$, that is, $x(T)=1$. Then $e^{T}=\left(x_{0}\right)^{1 / \lambda_{1}}$ and so

$$
x(T)=1, \quad y(T)=y_{0}\left(x_{0}\right)^{-\lambda_{2} / \lambda_{1}}, \quad z(T)=\left(x_{0}\right)^{-\lambda_{3} / \lambda_{1}} .
$$

Let $S=\{(x, y, 1):|x| \leq 1 / 2,|y| \leq 1 / 2\}$ be a transversal section to the field $Y$ such that the first return map $P$ is defined in $S^{*}=$ $S \backslash\{x=0\}$. The line $x=0$ in $S$ is contained in the intersection between $W^{s}(0, Y)$ and $S$. Let

$$
P: S^{*} \longrightarrow \operatorname{int}(S): p \mapsto P(p)
$$

be defined by $P(p)=Y_{\tau}(p)$, where $\tau$ is the first positive time such that $Y_{\tau}(p) \in S$. Assume the following hypothesis over the field $Y$ (for more details see [6, page 273]: 
(h1) The point $O$ has eigenvalues $\lambda_{1}, \lambda_{2}, \lambda_{3}$ such that $0<-\lambda_{3}<\lambda_{1}<$ $-\lambda_{2}$, where $\lambda_{3}$ is the eigenvalue of the $z$-axis, which is supposed to be $Y_{t}$-invariant.

(h2) There exists a foliation $\mathcal{F}^{s}$ of $S$ whose vertical leaves are such that: if $L \in \mathcal{F}^{s}$ and $P$ is defined in $L$, then $P(L)$ is contained in a leaf of $\mathcal{F}^{s}$. The foliation $\mathcal{F}^{s}$ is part of the strong stable manifold of the flow in the attractor which can be extended to a neighborhood of the attractor [11].

(h3) Every point of $S^{*}$ returns to $S$, and the return map $P$ is expansive enough in the direction which is transverse to the leaves of $\mathcal{F}^{s}$.

(h4) The flow is symmetric with respect to the rotation $\theta=\pi$ around the $y$-axis.

These four hypothesis define the geometric Lorenz flow. Analytically, these hypothesis may be reformulated by a coordinate system $(x, y)$ over $S$ such that $P$ has the following properties:

(P1) The leaves of $\mathcal{F}^{s}$ are given by $x=c$, with $-1 / 2 \leq x \leq 1 / 2$.

(P2) There are functions $f$ and $g$ such that $P$ has the form:

$$
P(x, y)=(f(x), g(x, y))
$$

for $x \neq 0$ and $P(-x,-y)=-P(x, y)$.

(P3) $f^{\prime}(x) \geq \lambda>\sqrt{2}$, for all $x \neq 0$ and $\lim _{x \rightarrow 0} f^{\prime}(x)=\infty$.

(P4) $0<\frac{\partial g}{\partial y}<\delta<1$, for all $x \neq 0$ and $\lim _{x \rightarrow 0} \frac{\partial g}{\partial y}=0$.

3.2. Foliations. In the text, the leaves of the foliation $\mathcal{F}^{s}$, of hypothesis (h2), will be identified to the lines in $S$ whose $x$-coordinate is fixed. For simplicity we will denote such a foliation by $\mathcal{F}$ and its leaves by

$$
\mathcal{F}_{x_{0}}=\left\{\left(x_{0}, y, 1\right) \in \mathbb{R}^{3} \mid y \in\left[-\frac{1}{2}, \frac{1}{2}\right]\right\} .
$$

Additionally, we will denote by

$$
L^{-}=\mathcal{F}_{-1 / 2}, \quad L_{0}=\mathcal{F}_{0}, \quad L^{+}=\mathcal{F}_{1 / 2} .
$$


We call $L_{0}$ of singular leaf and denote $\mathcal{F}^{*}=\mathcal{F} \backslash L_{0}$. The onedimensional map $f,(\mathrm{P} 2)$, induces a map $\widehat{f}$ given by

$$
\begin{aligned}
\widehat{f}: \mathcal{F}^{*} & \longrightarrow \mathcal{F} \\
\mathcal{F}_{x} & \longmapsto \mathcal{F}_{f(x)} .
\end{aligned}
$$

Whenever there is no ambiguity, we shall denote $\widehat{f}$ by $f$.

We define an order relation on $\mathcal{F}$ by:

$$
\mathcal{F}_{x} \leq \mathcal{F}_{y} \Longleftrightarrow x \leq y
$$

where $x, y \in[-1 / 2,1 / 2]$.

4. Proof of Theorem 1.1. The proof of Theorem 1.1 will be developed in the next three subsections. In Section 4.1, we define a pseudo orbit. In Section 4.2, we prove some technical lemmas. Lastly, in Section 4.3, we prove the theorem itself.

From now on, $(\Lambda, \varphi)$ is a geometric Lorenz flow, $P: S^{*} \rightarrow S$ is the associated Poincaré map and $f: \mathcal{F}^{*} \rightarrow \mathcal{F}$ is the map associated to the foliation $\mathcal{F}$ over $S$.

4.1. The pseudo orbit. This section concerns the description of the pseudo orbit to be used in the proof of the Theorem 1.1. We will develop it under the assumption that

$$
f\left(L^{-}\right)>L^{-} \quad \text { and } \quad f\left(L^{+}\right)=L^{+}
$$

(recall the order relation on the set of leaves of $S$ ). The remainder case, which is $f\left(L^{-}\right) \geq L^{-}$and $f\left(L^{+}\right)<L^{+}$, will be commented in Remark 4.2 (subsection 4.1.3).

Recall that a pseudo orbit is a bi-sequence (when dealing with flows) composed by points and times. We are going to separately construct two sequences. Firstly, the sequence of points and then the sequence of times. Finally, we will argue that such a sequence is, in fact, a pseudo orbit in the sense of the three kinds of pseudo orbit defined in Section 2.

4.1.1. Sequence of points. Denote by $V$ the set contained in the stable manifold of the singularity $\sigma=(0,0,0)$ and "under" the singular leaf $L_{0}$ 
of $S$ (see Figure 4), namely,

$$
V=\bigcup_{x \in L_{0}} \varphi\left(\mathbb{R}^{+}, x\right) .
$$

The positive orbit of points in $V$ converges to $\sigma$; thus, they do not cross the section $S$. In turn, all the other points cross $S$ in the future. Now, for any $x \in U \backslash V$, let $\tau(x)$ be the time spent by the flow to intersect $S$,

$$
\tau(x)=\min \left\{t \in \mathbb{R}_{*}^{+} \mid \varphi(t, x) \in S\right\},
$$

where $\mathbb{R}_{*}^{+}$denotes the set of positive real numbers, and let $\pi(x)$ be such an intersection point

$$
\pi(x)=\varphi(\tau(x), x)
$$

Put

$$
\pi^{0}(x)=x \quad \text { and } \quad \pi^{n}(x)=\pi\left(\pi^{n-1}(x)\right) \text { for all } n>0 .
$$

Fix a constant $\Gamma$ much smaller than the distance between the lateral leaves:

$$
\Gamma \ll d\left(L^{-}, L^{+}\right)
$$

(here $d(A, B)=\inf \{d(x, y) \mid x \in A$ and $y \in B\}$ for any sets $A$ and $B$ ). We will construct a one-sided sequence $\left(x_{n}\right)_{n \in \mathbb{N}}$ in four steps. In the construction, we assume that $k$ is an arbitrary natural number or zero.

Step 1. The terms of the sequence of type $x_{4 k}$ are points in $W^{u,-}(\sigma)$ which verify

$$
d\left(x_{4 k}, \sigma\right)=\frac{\Gamma}{\sqrt{2} \cdot 2^{k}}
$$

(see Figure 2).

Step 2. In the terms of type $x_{4 k+1}$ we will impose two conditions. From the above item, we have defined the term $x_{0}$ and we also have $\pi\left(x_{4 k}\right)=\pi\left(x_{0}\right)$. So, we take $x_{4 k+1} \in S^{+}$satisfying a first condition

$$
d\left(x_{4 k+1}, \pi\left(x_{0}\right)\right)=\frac{\Gamma}{2^{k}} .
$$

Now, the positive orbit of any point in the set $U_{i=1}^{\infty} f^{-i}\left(L_{0}\right)$ intersects $L_{0}$. In turn, such a set is dense in $S$. Based on this fact, we impose a 
second condition on $x_{4 k+1}$ :

$$
\varphi\left(s_{k}^{1}, x_{4 k+1}\right) \in L_{0} \quad \text { for some } s_{k}^{1}>1 .
$$

Note that, naturally, we have

$$
\mathcal{F}_{x_{4 k+1}}<L^{+}=\mathcal{F}_{\pi\left(x_{0}\right)} .
$$

Step 3. Similarly as in Step 1, we take $x_{4 k+2} \in W^{u,+}(\sigma)$, verifying

$$
d\left(x_{4 k+2}, \sigma\right)=\frac{\Gamma}{\sqrt{2} \cdot 2^{k}} .
$$

Step 4. Finally, the terms of type $x_{4 k+3}$ belong to $S^{-}$and we will demand three conditions for them. Before this, note that $\pi\left(x_{4 k+2}\right)=$ $\pi\left(x_{2}\right)$ (and thus $\pi^{2}\left(x_{4 k+2}\right)=\pi^{2}\left(x_{2}\right)$ ), and we can reduce $\Gamma$ such that $d\left(\pi\left(L^{-}\right), L^{-}\right)>\Gamma / 2^{k}$. So, the first two conditions are:

$$
d\left(x_{4 k+3}, \pi^{2}\left(x_{2}\right)\right)=\frac{\Gamma}{2^{k}}
$$

and

$$
\varphi\left(s_{k}^{3}, x_{4 k+3}\right) \in L_{0} \quad \text { for some } s_{k}^{3}>1 \text {. }
$$

In addition, note that $\mathcal{F}_{\pi\left(x_{4 k+2}\right)}=L^{-}$and $\mathcal{F}_{\pi^{2}\left(x_{4 k+2}\right)}=f\left(L^{-}\right)$. Moreover, we know that $f\left(L^{-}\right)>L^{-}$. These facts allow us to choose $x_{4 k+3} \in S^{-}$satisfying the third condition:

$$
\mathcal{F}_{\pi\left(x_{4 k+2}\right)}=L^{-}<\mathcal{F}_{x_{4 k+3}}<f\left(L^{-}\right)=\mathcal{F}_{\pi^{2}\left(x_{4 k+2}\right)} .
$$

Now we extend the above sequence to a two-sided sequence $\left(x_{n}\right)_{n \in \mathbb{Z}}$ by setting

$$
x_{4 k+i}=x_{4(-k-1)+i},
$$

for $k \leq-1$ and $i \in\{0, \ldots, 3\}$. Moreover, the expressions

$$
\frac{\Gamma}{2^{k}} \text { and } \frac{\Gamma}{\sqrt{2} \cdot 2^{k}}
$$

in the above equalities must be replaced, respectively, by

$$
\frac{\Gamma}{2^{(-k)}} \text { and } \frac{\Gamma}{\sqrt{2} \cdot 2^{(-k)}} .
$$

This ends the construction of the sequence of points. 
4.1.2. Sequence of times. In the same way as the last subsection we will construct a sequence of times $\left(t_{n}\right)_{n \in \mathbb{N}}$ in four steps. In the construction we assume that $k$ is an arbitrary natural number or zero.

Step 1. The terms of type $t_{4 k}$ are the times that the points $x_{4 k}$ spend to reach $\pi\left(x_{4 k}\right) \in S$, namely:

$$
t_{4 k}=\tau\left(x_{4 k}\right) .
$$

Step 2. Recall that $x_{4 k+1}$ spends a time $s_{k}^{1}$ (through the flow) to reach the point $\varphi\left(s_{k}^{1}, x_{4 k+1}\right)$ in the singular leaf $L_{0}$. In turn, such a point tends to the singularity. So there exists a time $\widetilde{s}_{k}^{1}$, verifying

$$
d\left(\varphi\left(s_{k}^{1}+\tilde{s}_{k}^{1}, x_{4 k+1}\right), \sigma\right)=\frac{\Gamma}{\sqrt{2} \cdot 2^{k}} .
$$

Therefore, we define the terms of type $t_{4 k+1}$ as

$$
t_{4 k+1}=s_{k}^{1}+\widetilde{s}_{k}^{1} \text {. }
$$

Step 3. The terms of type $t_{4 k+2}$ are the times that the points $x_{4 k+2}$ spend to reach $\pi^{2}\left(x_{4 k+2}\right) \in S$, namely:

$$
t_{4 k+2}=\tau\left(x_{4 k+2}\right)+\tau\left(\pi\left(x_{4 k+2}\right)\right) .
$$

Step 4. As in item (iii), $s_{k}^{3}$ is a time such that $\varphi\left(s_{k}^{3}, x_{4 k+3}\right)$ lies in the singular leaf $L_{0}$. So there is $\widetilde{s}_{k}^{3}$ satisfying

$$
d\left(\varphi\left(s_{k}^{3}+\widetilde{s}_{k}^{3}, x_{4 k+3}\right), \sigma\right)=\frac{\Gamma}{\sqrt{2} \cdot 2^{k}} .
$$

Therefore, we define the terms of type $t_{4 k+3}$ as

$$
t_{4 k+3}=s_{k}^{3}+\widetilde{s}_{k}^{3} .
$$

Now we extend the above sequence to a two-sided sequence $\left(t_{n}\right)_{n \in \mathbb{Z}}$ by setting

$$
t_{4 k+i}=t_{4(-k-1)+i}
$$

for $k \leq-1$ and $i \in\{0, \ldots, 3\}$. As in (4.8), we replace $k$ by $-k$. This ends the construction of the sequence of times. 
4.1.3. The pseudo orbit.

Definition 4.1. Let

$$
\left(x_{n}, t_{n}\right)_{n \in \mathbb{Z}}
$$

be the bi-sequence whose component sequence $\left(x_{n}\right)_{n \in \mathbb{Z}}$ is the sequence of points constructed in subsection 4.1.1 and the component sequence $\left(t_{n}\right)_{n \in \mathbb{Z}}$ is the sequence of times constructed in subsection 4.1.2.

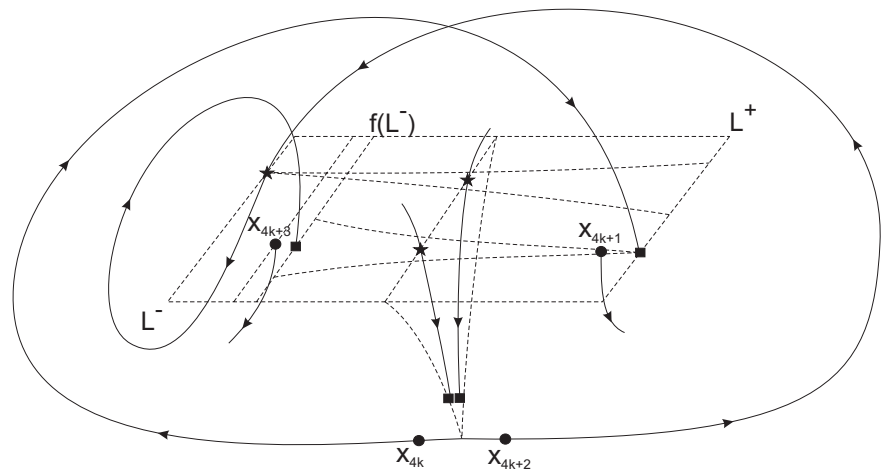

FIGURE $2 . \quad k \geq 0$. The points in shape of circle compose the pseudo orbit. Square points: in $L^{+}$is $\pi\left(x_{4 k}\right)$, in $f\left(L^{-}\right)$is $\pi^{2}\left(x_{4 k+2}\right)$, near the origin at right is $\varphi\left(t_{4 k+1}, x_{4 k+1}\right)$, and at left is $\varphi\left(t_{4 k+3}, x_{4 k+3}\right)$. Star points: in the singular leaf at right is $\varphi\left(s_{k}^{1}, x_{4 k+1}\right)$, at left is $\varphi\left(s_{k}^{3}, x_{4 k+3}\right)$ and in $L^{-}$is $\pi\left(x_{4 k+2}\right)$.

Remark 4.2. To the case

$$
f\left(L^{-}\right)=L^{-} \quad \text { and } \quad f\left(L^{+}\right)<L^{+}
$$

we set another bi-sequence, say $\left(\bar{x}_{n}, \bar{t}_{n}\right)_{n \in \mathbb{Z}}$, whose construction is the same of (4.13), up to demanding

$$
\mathcal{F}_{\pi^{2}\left(x_{4 k}\right)}=f\left(L^{+}\right)<\mathcal{F}_{x_{4 k+1}}<L^{+}=\mathcal{F}_{\pi\left(x_{0}\right)},
$$

instead of (4.4), and

$$
L^{-}=\mathcal{F}_{\pi\left(x_{4 k+2}\right)}<\mathcal{F}_{x_{4 k+3}}
$$


instead of (4.6) (the construction of the sequence of times does not change).

Additionally, to the case

$$
f\left(L^{-}\right)>L^{-} \quad \text { and } \quad f\left(L^{+}\right)<L^{+}
$$

we order as in (4.14) and (4.6).

The proofs of the further results are done only to the bi-sequence $\left(x_{n}, t_{n}\right)_{n \in \mathbb{Z}}$. In Lemma 4.6 , the analyzed cases are symmetrically the same. The remaining facts (which deal only with distances) are verbatim.

Proposition 4.3. The bi-sequence

$$
\left(x_{n}, t_{n}\right)_{n \in \mathbb{Z}}
$$

given by Definition 4.1, is a $\delta$-average-pseudo orbit for any $\delta>0$. Moreover, it is also a limit-pseudo orbit and an asymptotic averagepseudo orbit.

Proof. Define a (positive) sequence $\left(A_{m}\right)_{m \in \mathbb{Z}}$ by

$$
A_{m}=d\left(\varphi\left(t_{m}, x_{m}\right), x_{m+1}\right) .
$$

Firstly we claim that

$$
A_{4 k+i}=\frac{\Gamma}{2^{|k|}}
$$

for any $k \in \mathbb{Z}$ and any $i \in\{0,1,2,3\}$ (see Figure 3 ).

Indeed, suppose that $k \geq 0$. Then

(i)

$$
\begin{aligned}
d\left(\varphi\left(t_{4 k}, x_{4 k}\right), x_{4 k+1}\right) & \stackrel{(4.9)}{=} d\left(\varphi\left(\tau\left(x_{4 k}\right), x_{4 k}\right), x_{4 k+1}\right) \\
& \stackrel{(4.1)}{=} d\left(\pi\left(x_{4 k}\right), x_{4 k+1}\right) \stackrel{(4.3)}{=} \frac{\Gamma}{2^{k}} .
\end{aligned}
$$




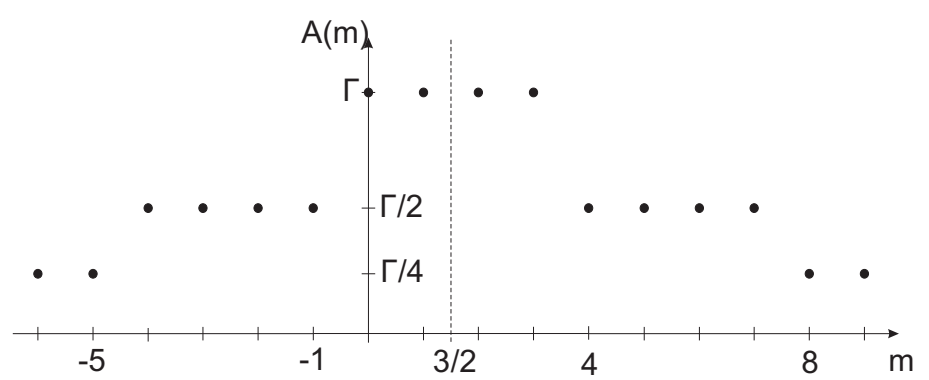

Figure 3. Graphic of the sequence $A_{m}$.

(ii)

$$
\begin{aligned}
& d\left(\varphi\left(t_{4 k+1}, x_{4 k+1}\right), x_{4 k+2}\right)=\sqrt{\left[d\left(\varphi\left(t_{4 k+1}, x_{4 k+1}\right), \sigma\right)\right]^{2}+\left[d\left(\sigma, x_{4 k+2}\right)\right]^{2}} \\
& \stackrel{(4.11)}{=} \sqrt{\left[d\left(\varphi\left(s_{k}^{1}+\widetilde{s}_{k}^{1}, x_{4 k+1}\right), \sigma\right)\right]^{2}+\left[d\left(\sigma, x_{4 k+2}\right)\right]^{2}} \\
& \stackrel{(4.10),(4.5)}{=} \sqrt{\left[\frac{\Gamma}{\sqrt{2} .2^{k}}\right]^{2}+\left[\frac{\Gamma}{\sqrt{2} .2^{k}}\right]^{2}}=\frac{\Gamma}{2^{k}} .
\end{aligned}
$$

The cases whose subscript indices are $4 k+2$ and $4 k+3$ are analogous to (i) and (ii), respectively. Summarizing, we have

$$
d\left(\varphi\left(t_{4 k+i}, x_{4 k+i}\right), x_{4 k+i}=\frac{\Gamma}{2^{k}},\right.
$$

for $k \geq 0$ and $i \in\{0,1,2,3\}$. On the other hand, following the same procedure, one can use the conversion formulas (4.7), (4.8) and (4.12), to prove that

$$
d\left(\varphi\left(t_{4 k+i}, x_{4 k+i}\right), x_{4 k+i}\right)=\frac{\Gamma}{2^{(-k)}},
$$

for $k \leq-1$ and $i \in\{0,1,2,3\}$. This proves our claim.

From (4.16), we conclude that:

(a)

$$
\lim _{|m| \rightarrow \infty} A_{m}=0
$$


that is, the sequence $\left(x_{n}, t_{n}\right)_{n \in \mathbb{Z}}$ is a limit-pseudo orbit.

Now we will verify that the sequence $\left(x_{n}, t_{n}\right)_{n \in \mathbb{Z}}$ is an asymptotic average-pseudo orbit. In fact,

$$
\begin{aligned}
\sum_{j=-n}^{n} d\left(\varphi\left(t_{j}, x_{j}\right), x_{j+1}\right) & \leq \sum_{j=0}^{2 n} d\left(\varphi\left(t_{j}, x_{j}\right), x_{j+1}\right) \\
& \leq 4 \sum_{j=0}^{2 n} d\left(\varphi\left(t_{4 j}, x_{4 j}\right), x_{4 j+1}\right) \\
& \leq 4 \sum_{j=0}^{2 n} \frac{\Gamma}{2^{j}}
\end{aligned}
$$

Therefore,

$$
\lim _{n \rightarrow \infty} \frac{1}{n} \sum_{j=-n}^{n} d\left(\varphi\left(t_{j}, x_{j}\right), x_{j+1}\right)=0
$$

Finally, we are going to show that the bi-sequence $\left(x_{n}, t_{n}\right)_{n \in \mathbb{Z}}$ is a $\delta$-average-pseudo orbit for any $\delta>0$. Given $\delta>0$, then we have, by (4.17), that there exists $N>0$ such that, for all $n>N$ and all $u \in Z$, we have:

$$
\frac{1}{n} \sum_{i=1}^{n} d\left(\varphi\left(t_{i+u}, x_{i+u}\right), x_{i+u+1}\right) \leq \frac{1}{n} \sum_{i=-n}^{n} d\left(\varphi\left(t_{i}, x_{i}\right), x_{i+1}\right)<\delta .
$$

4.2. Technical lemmas. In this section, we are going to prove two technical lemmas. Before that, let us define an object and review some notation.

Let $U$ be an isolating block of geometric Lorenz attractors (Section $3)$. For any fixed point $y$ in $U$, the function $t \mapsto \varphi(h(t), y)$ is continuous (because the flow is smooth and $h$ is a homeomorphism). Hence, from the compactness of manifold $M$ we can get the following definition.

Definition 4.4. Let $h$ be a reparametrization in $\operatorname{Rep}(\Delta)$. Then $\beta$ is a positive real constant such that

$$
d(\varphi(h(t), y), y)<\frac{\Gamma}{2}
$$


for all $t \in[-\beta, \beta]$ and all $y \in U$.

Recall that, if $\left(z_{n}, t_{n}\right)_{n \in \mathbb{Z}}$ is an arbitrary bi-sequence, then for any $n \in \mathbb{N}$, we denote by

$$
s_{n}=\sum_{i=0}^{n-1} t_{i} \quad \text { and } \quad s_{-n}=\sum_{i=-n}^{-1} t_{i} .
$$

Additionally, we set $s_{0}=0$.

Lemma 4.5. Let $\left(x_{n}, t_{n}\right)_{n \in \mathbb{Z}}$ be the sequence (4.15). Take a point $y$ in $U$, a reparametrization $h$ in $\operatorname{Rep}(\Delta)$ and an integer number $k$. If

$$
d\left(\varphi(h(t), y), \varphi\left(t-s_{4 k+u}, x_{4 k+u}\right)\right)<2 \Gamma
$$

for any $u \in\{0, \ldots, 3\}$ and all $t \in\left[s_{4 k+u}, s_{4 k+u+1}-\beta\right)$, then

$$
d\left(\varphi(h(t), y), \varphi\left(t-s_{4 k+u}, x_{4 k+u}\right)\right)<3 \Gamma
$$

for any $u \in\{0, \ldots, 3\}$ and all $t \in\left[s_{4 k+u}, s_{4 k+u+1}\right)$.

Proof. Fix $u \in\{0, \ldots, 3\}$. We are going to verify the inequality (4.20) for any $t \in\left[s_{4 k+u}, s_{4 k+u+1}\right)$.

If

$$
t \in\left[s_{4 k+u}, s_{4 k+u+1}-\beta\right),
$$

then we get (4.20) directly from (4.19).

Now we will analyze the remainder case:

$$
t \in\left[s_{4 k+u+1}-\beta, s_{4 k+u+1}\right) .
$$

We will proceed by contradiction. Assume that there exist $t_{0} \in$ $\left[s_{4 k+u+1}-\beta, s_{4 k+u+1}\right)$ for which the inequality (4.20) does not hold, that is,

$$
d\left(\varphi\left(h\left(t_{0}\right), y\right), \varphi\left(t_{0}-s_{4 k+u}, x_{4 k+u}\right)>3 \Gamma .\right.
$$

To shorter the expressions, we will denote by

$$
p(t)=\varphi(h(t), y)
$$

and

$$
q(t)=\varphi\left(t-s_{4 k+u}, x_{4 k+u}\right)
$$


for $t \in \mathbb{R}$. Then

$$
\begin{aligned}
d\left(p\left(t_{0}-\beta\right), q\left(t_{0}-\beta\right)\right)> & d\left(q\left(t_{0}\right), p\left(t_{0}-\beta\right)\right)-d\left(q\left(t_{0}\right), q\left(t_{0}-\beta\right)\right), \\
> & {\left[d\left(p\left(t_{0}\right), q\left(t_{0}\right)\right)-d\left(p\left(t_{0}\right), p\left(t_{0}-\beta\right)\right)\right] } \\
& -d\left(q\left(t_{0}\right), q\left(t_{0}-\beta\right)\right), \\
& \stackrel{(4.21)}{>} 3 \Gamma-\left[d\left(p\left(t_{0}\right), p\left(t_{0}-\beta\right)\right)+d\left(q\left(t_{0}\right), q\left(t_{0}-\beta\right)\right)\right], \\
& \stackrel{(4.18)}{>} 3 \Gamma-\left(\frac{\Gamma}{2}+\frac{\Gamma}{2}\right)=2 \Gamma .
\end{aligned}
$$

Summarizing, we have

$$
d\left(\varphi\left(h\left(t_{0}-\beta\right), y\right) \varphi\left(\left(t_{0}-\beta\right)-s_{4 k+u}, x_{4 k+u}\right)\right)>2 \Gamma .
$$

On the other hand, note that the time $t_{0}-\beta$ belongs to the interval $\left[s_{4 k+u}, s_{4 k+u+1}-\beta\right)$. This contradicts the inequality (4.19) of our hypothesis. This contradiction ends the proof.

Given a real time $t$, then we can associate the following point in $U$ :

$$
\varphi\left(t-s_{n}, x_{n}\right)
$$

where $n$ is such that $t \in\left[s_{n}, s_{n+1}\right)$. From now on, we will say the pseudo orbit $\left(x_{n}, t_{n}\right)_{n \in \mathbb{Z}}$, as a reference to all the points in $M$ obtained by the above association. We will also write the pseudo orbit $\left(x_{n}, t_{n}\right)_{n \in \mathbb{Z}}$ instead of the sequence $\left(x_{n}, t_{n}\right)_{n \in \mathbb{Z}}$.

If necessary, we can reduce the size of $\Gamma$ (see (4.2)) in order to have

$$
d\left(V, \Sigma^{-}\right)>3 \Gamma \quad \text { and } \quad d\left(V, \Sigma^{+}\right)>3 \Gamma .
$$

(see Figure 4). From the above inequalities and the definition of the regions $U^{+}$and $U^{-}$, we also get

$$
d\left(\Sigma^{+}, U^{-}\right)>3 \Gamma \quad \text { and } \quad d\left(\Sigma^{-}, U^{+}\right)>3 \Gamma .
$$

Lemma 4.6. Let $\left(x_{n}, t_{n}\right)_{n \in \mathbb{Z}}$ be the pseudo orbit (4.15). Take a point $y$ in $U$, a reparametrization $h$ in $\operatorname{Rep}(\Delta)$. Then, for each number $k \in \mathbb{Z}$, there exist a number

$$
u \in\{0, \ldots, 4\}
$$

and a time

$$
t \in\left[s_{4 k+u}, s_{4 k+u+1}-\beta\right)
$$




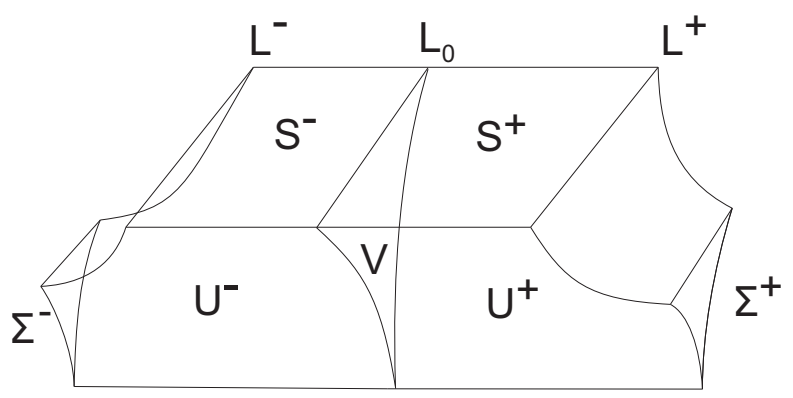

FiguRE 4. Regions.

such that the following holds

$$
d\left(\varphi(h(t), y), \varphi\left(t-s_{4 k+u}, x_{4 k+u}\right)\right) \geq 2 \Gamma .
$$

Proof. Fix $k \in \mathbb{Z}$. The proof will follow by exclusion: suppose that there is no number $u \in\{0, \ldots, 3\}$ and no time $t \in\left[s_{4 k+u}, s_{4 k+u+1}-\beta\right)$ for which the inequality (4.24) is verified. Then we are going to exhibit a time $t_{0}$ inside the interval $\left[s_{4 k+4}, s_{4 k+5}-\beta\right)$ satisfying

$$
d\left(\varphi\left(h\left(t_{0}\right), y\right), \varphi\left(t_{0}-s_{4 k+4}, x_{4 k+4}\right)\right)>2 \Gamma .
$$

Such an exhibition concludes the proof of this lemma.

Before we begin with the rest of the proof let us recall some definitions. If $z$ is a point in $U$, then $\tau(z)$ is the time spent by the flow through $z$ to reach the cross section $S ; \pi(z)$ is such an intersection point; $\pi^{n}(z)=\pi\left(\pi^{n-1}(z)\right)$, and $\mathcal{F}_{\pi(z)}$ is the leaf of the foliation $\mathcal{F}$ on $S$ containing the point $\pi(z)$.

Firstly, observe that, from our exclusion hypothesis at the beginning and Lemma 4.5, we have

$$
d\left(\varphi(h(t), y), \varphi\left(t-s_{4 k+u}, x_{4 k+u}\right)\right)<3 \Gamma
$$

for any $u \in\{0, \ldots, 3\}$ and all $t \in\left[s_{4 k+u}, s_{4 k+u+1}\right)$. In other words, for each time $t$ in the interval $\left[s_{4 k}, s_{4 k+4}\right)$, the point associated by the pseudo orbit $\left(x_{n}, t_{n}\right)_{n \in \mathbb{Z}}$ and the point associated by the (reparameterized) orbit through $y$ are at a distance less than $3 \Gamma$. Therefore, as the time in such an interval goes forward, the orbit and the pseudo orbit 
intersect the cross section $S$ the same number of times. In particular, the points $\pi(y)$ and $x_{4 k+1}$ are close.

From the definition of the terms of type $x_{4 k+1}$, there exists a number $m \in \mathbb{N}$ such that the point $\pi^{m}\left(x_{4 k+1}\right)$ lies on the singular leaf $L_{0}$. Recall that the cross section $S$ is a (disjoint) union of the leaf $L_{0}$ with the "pieces" $S^{-}$and $S^{+}$.

Now we suppose that there exists a number $j \in\{0, \ldots, m-1\}$ for which the points $\pi^{j}(\pi(y))$ and $\pi^{j}\left(x_{4 k+1}\right)$ are in distinct pieces, namely,

$$
\pi^{j}(\pi(y)) \in S^{+} \Longrightarrow \pi^{j}\left(x_{4 k+1}\right) \in S^{-}
$$

or

$$
\pi^{j}(\pi(y)) \in S^{-} \Longrightarrow \pi^{j}\left(x_{4 k+1}\right) \in S^{+} .
$$

Let us proceed with our argument using the implication (4.27) (the usage of (4.28) would be symmetrical). In this case, there exists a time $t_{0} \in\left[s_{4 k+1}, s_{4 k+2}\right)$ such that

- either

$$
\varphi\left(h\left(t_{0}\right), y\right) \in \Sigma^{+} \quad \text { and } \quad \varphi\left(t_{0}-s_{4 k+1}, x_{4 k+1}\right) \in U^{-}
$$

- or

$$
\varphi\left(t_{0}-s_{4 k+1}, x_{4 k+1}\right) \in \Sigma^{-} \text {and } \varphi\left(h\left(t_{0}\right), y\right) \in U^{+} .
$$

In the first case, we have

$$
\left.d\left(\varphi\left(t_{0}\right), y\right), \varphi\left(t_{0}-s_{4 k+1}, x_{4 k+1}\right)\right) \geq d\left(\Sigma^{+}, U^{-}\right) \stackrel{(4.23)}{>} 3 \Gamma .
$$

In the second case, we have

$$
\left.d\left(\varphi\left(t_{0}\right), y\right), \varphi\left(t_{0}-s_{4 k+1}, x_{4 k+1}\right)\right) \geq d\left(U^{+}, \Sigma^{-}\right) \stackrel{(4.23)}{>} 3 \Gamma .
$$

In both cases we fall into contradiction with (4.26). So, this contradiction means that the points $\pi^{j}(\pi(y))$ and $\pi^{j}\left(x_{4 k+1}\right)$ are always in the same piece, namely:

$$
\pi^{j}(\pi(y)) \in S^{ \pm} \Longrightarrow \pi^{j}\left(x_{4 k+1}\right) \in S^{ \pm}
$$

for all $j \in\{0, \ldots, m-1\}$.

Now consider the leaves $\mathcal{F}_{\pi(y)}$ and $\mathcal{F}_{x_{4 k+1}}$ of the foliation $\mathcal{F}$ defined on $S$. Recall that we defined an order relation " $\leq$ " on $\mathcal{F}$. We will finish 
the proof of this lemma by analyzing such an order relation over these leaves, $\mathcal{F}_{\pi(y)}$ and $\mathcal{F}_{x_{4 k+1}}$.

Case (1): $\mathcal{F}_{\pi(y)}>\mathcal{F}_{x_{4 k+1}}$. Observe that the functions $\left.f\right|_{S^{+}}$and $\left.f\right|_{S^{-}}$ are increasing. This fact, together with the implication (4.29) and the hypothesis of this case imply

$$
L_{0}=\mathcal{F}_{\pi^{m}\left(x_{4 k+1}\right)}<\mathcal{F}_{\pi^{m+1}(y)},
$$

(see Figure 5). Thus,

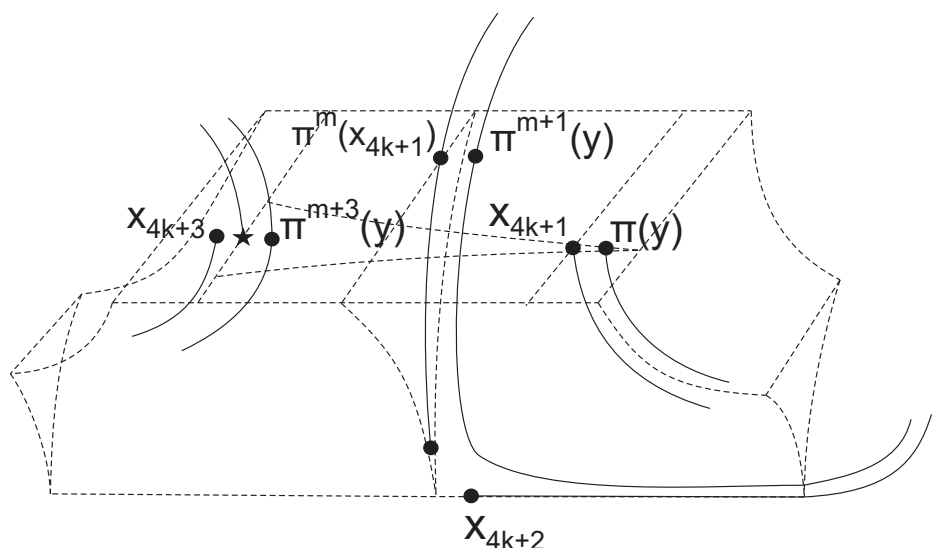

Figure 5. The orbit and the pseudo orbit are still close for times in $\left[s_{4 k}, s_{4 k+4}\right)$. The star point is $\pi^{2}\left(x_{4 k+2}\right)$.

$$
\mathcal{F}_{\pi\left(x_{4 k+2}\right)}=L^{-}<\mathcal{F}_{\pi^{m+2}(y)}<L_{0}
$$

Hence,

$$
\mathcal{F}_{x_{4 k+3}} \stackrel{(4.6)}{<} \mathcal{F}_{\pi^{2}\left(x_{4 k+2}\right)}<\mathcal{F}_{\pi^{m+3}(y)}
$$

From the definition of the terms of type $x_{4 k+3}$, there exists a number $q \in \mathbb{N}$ such that the point $\pi^{q}\left(x_{4 k+3}\right)$ lies on the singular leaf $L_{0}$. From the same argument we used to conclude the implication (4.29) (that the orbit and the pseudo orbit must hit $S$ simultaneously in same pieces) 
we get

$$
\mathcal{F}_{\pi^{j}\left(x_{4 k+3}\right)} \in S^{ \pm} \Longrightarrow \mathcal{F}_{\pi^{j+m+3}(y)} \in S^{ \pm}
$$

for all $j \in\{0, \ldots, q-1\}$. Since the functions $\left.f\right|_{S^{+}}$and $\left.f\right|_{S^{-}}$are increasing, then, by (4.30) and (4.31), we have

$$
L_{0}=\mathcal{F}_{\pi^{q}\left(x_{4 k+3}\right)}<\mathcal{F}_{\pi^{q+m+3}(y)} .
$$

Therefore,

- either

$$
\varphi\left(h\left(t_{0}\right), y\right) \in \Sigma^{+} \quad \text { and } \quad \varphi\left(t_{0}-s_{4 k+3}, x_{4 k+3}\right) \in V
$$

for some $t_{0} \in\left[s_{4 k+3}, s_{4 k+4}\right)$,

$$
\varphi\left(h\left(t_{0}\right), y\right) \in \Sigma^{+} \quad \text { and } \quad \varphi\left(t_{0}-s_{4 k+3}, x_{4 k+3}\right) \in U^{-}
$$

for some $t_{0} \in\left[s_{4 k+4}, s_{4 k+5}\right)$ (see Figure 6),

- or

$$
\varphi\left(t_{0}-s_{4 k+3}, x_{4 k+3}\right) \in \Sigma^{-} \text {and } \varphi\left(h\left(t_{0}\right), y\right) \in U^{+}
$$

for some $t_{0} \in\left[s_{4 k+4}, s_{4 k+5}\right)$.

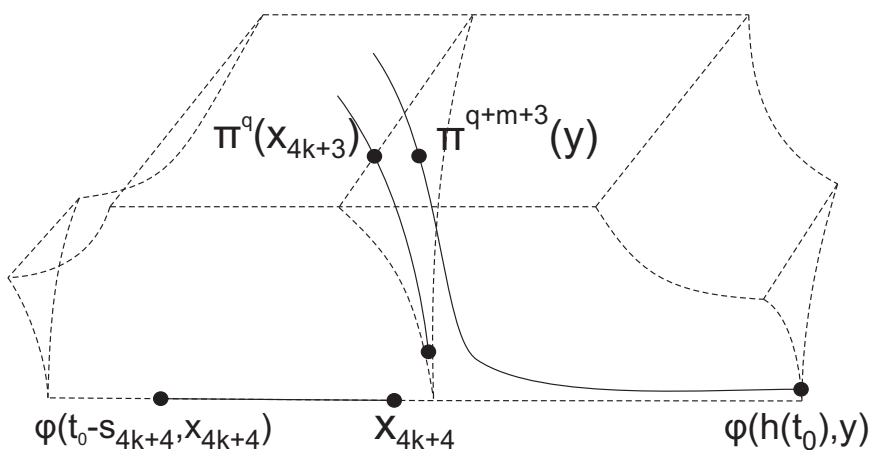

Figure 6. The orbit and the pseudo orbit gets far for a time $t_{0}$ in $\left[s_{4 k+4}, s_{4 k+5}-\beta\right)$.

The pertinent relations in (4.32) may not occur because, using (4.22), we can contradict (4.26). On the other hand, the two remaining 
conditions means that

$$
\left.d\left(\varphi\left(t_{0}\right), y\right), \varphi\left(t_{0}-s_{4 k+4}, x_{4 k+4}\right)\right) \stackrel{(4.23)}{>} 3 \Gamma>2 \Gamma
$$

for some $t_{0} \in\left[s_{4 k+4}, s_{4 k+5}\right)$. This is almost what we wanted to show (see $(4.25))$.

Recall the definition of $\beta$ on (4.18). We conclude the current case claiming that

$$
t_{0} \in\left[s_{4 k+4}, s_{4 k+5}-\beta\right) .
$$

In fact, the pseudo orbit through $x_{4 k+4}$ must cross $\Sigma^{-}$. This implies that there exists $\eta \geq 0$,

$$
\varphi\left(\left(t_{0}+\eta\right)-s_{4 k+4}, x_{4 k+4}\right) \in \Sigma^{-} .
$$

On the other hand, we have

$$
\varphi\left(s_{4 k+5}-s_{4 k+4}, x_{4 k+4}\right) \in S^{+} .
$$

Once $\overline{\Sigma^{-}}$and $\overline{S^{+}}$are disjoint, then the time

$$
s_{4 k+5}-\left(t_{0}+\eta\right)
$$

is bounded away from zero (uniformly on $k$ ). Hence, we can reduce $\beta$, if necessary, to get

$$
\beta<s_{4 k+5}-\left(t_{0}+\eta\right)
$$

So

$$
t_{0}<s_{4 k+5}-\beta
$$

as we wanted to show.

Case (2): $\mathcal{F}_{\pi(y)} \leq \mathcal{F}_{x_{4 k+1}}$. In this case, we have

$$
\mathcal{F}_{\pi^{m}(\pi(y))} \leq \mathcal{F}_{\pi^{m}\left(x_{4 k+1}\right)}=L_{0} .
$$

Therefore, the orbit either gets into $V$ and stays there or gets into $U^{-}$and "escapes" through $\Sigma^{-}$. In turn, the pseudo orbit gets into $V$ and escapes through $\Sigma^{+}$. Anyway, we can use the same argument as in Case (1) to contradict the inequality (4.26). This means that this current case may not occur. The proof of the lemma is over.

4.3. Conclusion. In this section, we are going to prove the Theorem 1.1. 
Proof of Theorem 1.1. Firstly, we will prove that Lorenz flows $(\Lambda, \varphi)$ do not have the $\Delta$-asymptotic average shadowing property ( $\Delta$-AASP).

Our argument will be by contradiction: suppose that $(\Lambda, \varphi)$ has the $\Delta$-AASP. According to Definition 2.3, for any asymptotic averagepseudo orbit $\left(z_{n}, t_{n}\right)_{n \in \mathbb{Z}}$, there exist a point $y \in U$ and a reparametrization $h \in \operatorname{Rep}(\Delta)$ such that:

$$
\lim _{n \rightarrow \infty} \frac{1}{n} \sum_{i=0}^{n} \int_{s_{i}}^{s_{i+1}} d\left(\varphi(h(t), y), \varphi\left(t-s_{i}, z_{i}\right)\right) d t=0 .
$$

Our target is to contradict this last sentence.

Let $\left(x_{n}, t_{n}\right)_{n \in \mathbb{Z}}$ be the asymptotic average-pseudo orbit given by the Proposition 4.3. Take a point $y \in U$ and a reparametrization $h \in \operatorname{Rep}(\Delta)$. The procedure is as follows. We will find a subsequence of $\left(x_{n}, t_{n}\right)_{n \in \mathbb{Z}}$ such that the limit (4.33) will be strictly positive. This shall be enough to conclude the proof due to the arbitrariness of the point $y$ and the reparametrization $h$.

By Lemma 4.6, there exist sequences $\left(u_{k}\right)_{k \in \mathbb{Z}}$ and $\left(t_{k}\right)_{k \in \mathbb{Z}}$ verifying

$$
u_{k} \in\{0, \ldots, 4\}
$$

and

$$
t_{k} \in\left[s_{4 k+u_{k}}, s_{4 k+u_{k}+1}-\beta\right),
$$

for all $k \in \mathbb{Z}$ and such that the following holds:

$$
d\left(\varphi\left(h\left(t_{k}\right), y\right), \varphi\left(t_{k}-s_{4 k+u_{k}}, x_{4 k+u_{k}}\right)\right) \geq 2 \Gamma,
$$

for all $k \in \mathbb{Z}$.

Now we claim that

$$
d\left(\varphi\left(h\left(t_{k}+r\right), y\right), \varphi\left(\left(t_{k}+r\right)-s_{4 k+u_{k}}, x_{4 k+u_{k}}\right)\right)>\Gamma
$$

for all $r \in[-\beta, \beta]$.

In fact, one can verify this claim by using equation (4.35), the definition of $\beta$ (Definition 4.4) and the same argument as used in the proof of Lemma 4.5. In other words, this claim says that: the points $\varphi\left(h\left(t_{k}\right), y\right)$ and $\varphi\left(\left(t_{k}\right)-s_{4 k+u_{k}}, x_{4 k+u_{k}}\right)$, once $\Gamma$-far, spend a time larger than $\beta$ to be $\Gamma$-close again (if they do). 
Therefore, consider the following subsequence of $\left(x_{n}, t_{n}\right)_{n \in \mathbb{Z} \text { : }}$ :

$$
\left(x_{4 k+u_{k}}, t_{4 k+u_{k}}\right)_{k \in \mathbb{Z}} \text {. }
$$

Then

$$
\begin{gathered}
\lim _{k \rightarrow \infty} \frac{1}{4 k+u_{k}} \sum_{i=0}^{4 k+u_{k}} \int_{s_{i}}^{s_{i+1}} d\left(\varphi(h(t), y), \varphi\left(t-s_{i}, x_{i}\right) d t \geq\right. \\
\lim _{k \rightarrow \infty} \frac{1}{5 k} \sum_{i=1}^{k} \int_{s_{4 i+u_{i}}}^{s_{4 i+u_{i}+1}} d\left(\varphi(h(t), y), \varphi\left(t-s_{4 i+u_{i}}, x_{4 i+u_{i}}\right) d t \stackrel{(4.34)}{\geq}\right. \\
\lim _{k \rightarrow \infty} \frac{1}{5 k} \sum_{i=1}^{k} \int_{t_{i}}^{t_{i}+\beta} d\left(\varphi(h(t), y), \varphi\left(t-s_{4 i+u_{i}}, x_{4 i+u_{i}}\right) d t \stackrel{(4.36)}{\geq}\right. \\
\lim _{k \rightarrow \infty} \frac{1}{5 k} \sum_{i=1}^{k} \beta \Gamma=\frac{\beta \Gamma}{5},
\end{gathered}
$$

as we wanted to show.

In this second part we are going to argue about Lorenz flows not having the $\Delta$-average shadowing property $(\Delta$-ASP). The proof is done also by the indirect method: suppose that $(\Lambda, \varphi)$ has the $\Delta$ ASP. Then consider the same pseudo orbit as before, $\left(x_{n}, t_{n}\right)_{n \in \mathbb{Z}}$. By Proposition 4.3 , it is a $\delta$-average-pseudo orbit for every $\delta>0$. On the other hand,

$$
\lim _{n \rightarrow \infty} \frac{1}{n} \sum_{i=0}^{n} \int_{s_{i}}^{s_{i+1}} d\left(\varphi(h(t), y), \varphi\left(t-s_{i}, x_{i}\right)\right) d t>\frac{\beta \Gamma}{5},
$$

for any $y \in U$ and any $h \in \operatorname{Rep}(\Delta)$. So Definition 2.1 fails for every $\epsilon<\frac{\beta \Gamma}{5}$ (the limsup in (2.1) would be larger than $\epsilon$ ). This finishes the second part.

Finally, one can show that Lorenz flows $(\Lambda, \varphi)$ do not have the $\Delta$ LSP.

\section{REFERENCES}

1. V.S. Afraimovich, V.V. Bykov and L.P. Shil'nikov, On attracting structurally unstable limit sets of Lorenz attractor type, Trudy Mosk. Mat. Obsh. 44 (1982), 150-212. 
2. Vítor Araújo and Maria José Pacífico, Three-dimensional flows, Ergeb. Math. Grenz 3, Springer, Heidelberg, 2010.

3. M.L. Blank, Metric properties of minimal solutions of discrete periodical variational problems, Nonlinearity 2 (1989), 1-22.

4. Timo Eirola, Olavi Nevanlinna and Sergei Yu Pilyugin, Limit shadowing property, Num. Funct. Anal. Optim. 18 (1997), 75-92.

5. Rongbao $\mathrm{Gu}$, The asymptotic average-shadowing property and transitivity for flows, Chaos Solit. Fract. 41 (2009), 2234-2240.

6. John Guckenheimer and Philip Holmes, Nonlinear oscillations, dynamical systems, and bifurcations of vector fields, revised and corrected reprint of the 1983 original, Appl. Math. Sci. 42, Springer-Verlag, New York, 1990.

7. John Guckenheimer and R.F. Williams, Structural stability of Lorenz attractors, Inst. Hautes Études Sci. Publ. Math. 50 (1979), 59-72.

8. Shin Kiriki and Teruhiko Soma, Parameter-shifted shadowing property for geometric Lorenz attractors, Trans. Amer. Math. Soc. 357 (2005), 1325-1339 (electronic).

9. Motomasa Komuro, Lorenz attractors do not have the pseudo-orbit tracing property, J. Math. Soc. Japan 37 (1985), 489-514.

10. E.N. Lorenz, Deterministic non-periodic flow, J. Atmos. Sci. 20 (1963), $130-141$.

11. C. Robinson, Differentiability of the stable foliation of the model Lorenz equations, Lect. Notes Math. 898, Springer, Berlin, 1981.

12. Michael Shub, Global stability of dynamical systems, Springer-Verlag, New York, 1987.

13. Shlomo Sternberg, On the structure of local homeomorphisms of Euclidean n-space, II, Amer. J. Math. 80 (1958), 623-631.

14. R.F. Williams, The structure of Lorenz attractors, Lect. Notes Math. 615, Springer, Berlin, 1977.

15. Math. 50 (1979), 73-99.

16. James A. Yorke and Ellen D. Yorke, Metastable chaos: The transition to sustained chaotic behavior in the Lorenz model, J. Stat. Phys. 21 (1979), 263-277.

Instituto de Matemática, Universidade Federal do Rio de Janeiro, P.O. Box 68530, 21945-970, Rio DE JANEIRo, BraziL

Email address: arbieto@im.ufrj.br

Instituto de Matemática, Universidade Federal do Rio de Janeiro, P.O. Box 68530, 21945-970, Rio de JANEIRo, BraziL

Email address: joao_eduardo_reis@im.ufrj.br

Instituto de Matemática, Universidade Federal do Rio de Janeiro, P.O. Box 68530, 21945-970, Rio de Janeiro, BraziL

Email address: raquelribeiro@im.ufrj.br 